Pacific Journal of Mathematics

COMPLEMENTATION IN THE LATTICE OF REGULAR 


\section{COMPLEMENTATION IN THE LATTICE OF REGULAR TOPOLOGIES}

\section{JeAnetTe HuEBeneR}

The present paper is concerned with the lattice of regular topologies on a set, and establishes the following results: a complete, complemented sublattice of the lattice of regular topologies on a set is exhibited and shown to be anti-isomorphic to the lattice of equivalence relations on the set; the lattice of regular topologies on a set is shown to be nonmodular if the cardinality of the set is at least four; the problem of complementation for regular topologies is reduced to considering $T_{0}$ regular topologies without isolated points; conditions are found which are equivalent to a regular topology having a principal regular complement; then follow some conditions under which the problem can be reduced to considering connected spaces; the final section consists of constructions of complements for certain classes of regular topologies, which classes may or may not be exhaustive.

Principal regular topologies and relations. Let $(\mathscr{S}, \mathrm{V}, \Lambda)$ be the lattice of all topologies on a set $E . \quad(\mathscr{S}, \mathrm{V}, \Lambda)$ is complete, antiatomic, complemented, and, if $|E|$, the cardinality of $E$, is at least three, it is not modular, [10, pp. 384-5, 389-397]. Next, let $\left(\mathscr{R}, \bigvee, \Lambda^{r}\right)$ be the lattice of all regular topologies on $E .\left(\mathscr{R}, \mathrm{V}, \Lambda^{r}\right)$ is complete but not a sublattice of $(\mathscr{S}, \mathrm{V}, \Lambda)$. The greatest lower bound in $\mathscr{R}$ of a collection of topologies in $\mathscr{R}$ is only the least upper bound of all the regular topologies which are weaker than the collection's greatest lower bound in $\mathscr{S}$ [8, pp. 754-755].

The anti-atoms of $\mathscr{S}$ are the ultraspaces on $E$; these are topologies of the form $\mathfrak{S}(x, \mathscr{U})=P_{c}(x) \cup \mathscr{C}$ where $\mathscr{C}$ is an ultrafilter on $E$ different from $\mathscr{U}(x)=\{A \subset E: x \in A\}$ and where $P_{c}(x)=\{A \subset E: x \notin A\}$. Frohlich [5, p. 81, Satz 3] showed that every topology $\tau$ on $E$ is the infimum of the ultraspaces on $E$ which are finer than $\tau$.

The special sublattice of $(\mathscr{S}, \mathbf{V}, \Lambda)$, which is anti-isomorphic to the lattice of preorders on $E$, is called the lattice of principal topologies. From this sublattice Steiner [10, p. 383, Theorem 2.6; pp. 389-397] and van Rooij [16, p. 807] take their complements. Now an ultraspace is said to be principal if its topology is of the form $\mathfrak{S}(x, \mathscr{C}(y))$ where $x \neq y$. A topology $\tau$ is principal if $\tau=1$, or if $\tau$ is the infimum of the principal ultratopologies finer than $\tau$. These topologies are also characterized [10, pp. 381-2, Theorem 2.3] by the fact that they have a base of open sets which is minimal at each 
point, i.e. for any $x \in E$ every open set containing $x$ must contain the open set

$$
B_{x}=\{y \in E: \subseteq(x, \mathscr{U}(y)) \geqq \tau\} \text {. }
$$

(Throughout the paper $B_{x}$ in a principal topology $\sigma$ will denote the $\sigma$-open set minimal at the point $x$.) Using this characterization it is easily seen [10, p. 382, Theorem 2.5] that the principal topologies form a sublattice of $(\mathscr{S}, \mathrm{V}, \Lambda)$. The mapping establishing the antiisomorphism between this lattice and the lattice of preorders is given by

$$
\eta(\tau)=G_{\tau}=\{(x, y): \Im(x, \mathscr{U}(y)) \geqq \tau\}
$$

and

$$
\eta^{-1}(G)=\tau_{G}=\Lambda\{\subseteq(x, \mathscr{U}(p)):(x, y) \in G\} .
$$

In the lattice of regular topologies there is a sublattice of the lattice of principal topologies which has a familiar structure:

THEOREM 1.1. A principal topology $\tau$ on $E$ is regular iff its representation satisfies the condition $\mathfrak{S}(x, \mathscr{C}(y)) \geqq \tau$ implies $\mathfrak{S}(y$, $\mathscr{Q}(x)) \geqq \tau$ for any $x, y \in E$.

Proof. Suppose $\tau$ is principal and regular and that $\subseteq(x, \mathscr{U}(y)) \geqq$ $\tau$. Then $y \in B_{x}$ and $B_{y} \subset B_{x}$. Now $\sim B_{y}$ is a closed set not containing $y$; accordingly there exists $U \in \tau$ such that $U \supset \sim B_{y}$ and $U \cap B_{y}=\varnothing$ which implies that $U=\sim B_{y} \in \tau$. If $x \in \sim B_{y} \in \tau$, then $B_{y} \subset B_{x} \subset \sim B_{y}$ which is a contradiction. Hence $x \in B_{y}$ and $\mathfrak{S}(y, \mathscr{Q}(x)) \geqq \tau$.

Conversely, in terms of the base of minimal open sets, the condition, $\mathfrak{S}(x, \mathscr{C}(y)) \geqq \tau$ implies $\mathfrak{S}(y, \mathscr{C}(x)) \geqq \tau$ for any $x, y \in E$, become $y \in B_{x}$ iff $x \in B_{y}$. Hence $B_{x}=B_{y}$ or $B_{x} \cap B_{y}=\varnothing$ for every $x, y \in E$. In which case, if $U=\bigcup\left\{B_{y}: y \in U\right\} \in \tau$ and $x \in \sim U$ then $B_{x} \cap U=\varnothing$ and it follows that $\sim U=\bigcup\left\{B_{x}: x \in \sim U\right\} \in \tau$. Every open set being closed implies $\tau$ is regular.

COROLlaRY 1.2. A principal topology $\tau$ is regular iff $G_{\tau}$ is an equivalence relation.

That the lattice of equivalence relations is complemented is proven mot a mot as in Steiner [10, p. 389, Theorem 5.1].

CoRollary 1.3. The lattice of principal regular topologies on $E$ is a complete sublattice of $\left(\mathscr{R}, \mathbf{V}, \Lambda^{r}\right)$ and $(\mathscr{S}, \mathbf{V}, \mathbf{\Lambda})$. 
Finally, for $|E| \leqq 3$ the lattice $\left(\mathscr{R}, \mathrm{V}, \Lambda^{r}\right)$ is a modular sublattice of $(\mathscr{S}, \mathbf{V}, \Lambda)$. If $|E| \geqq 4$, then the lattice $\left(\mathscr{R}, \mathbf{V}, \Lambda^{r}\right)$ is not modular: Let $a, b, c, d$ be distinct points of $E$. Define each of the following principal regular topologies by its base of minimal open sets

$$
\begin{array}{cl}
\tau_{(a b)} & \{a, b\},\{c\},\{d\} \text { and }\{x\} \text { for } x \neq a, b, c, d \\
\tau_{(a b)(c d)} & \{a, b\},\{c, d\} \text { and }\{x\} \text { for } x \neq a, b, c, d \\
\tau_{(a d)(c b)} & \{a, d\},\{c, b\} \text { and }\{x\} \text { for } x \neq a, b, c, d \\
\tau_{(a b c d)} & \{a, b, c, d\} \text { and }\{x\} \text { for } x \neq a, b, c, d .
\end{array}
$$

Then we have the following diagram of least upper bounds and greatest lower bounds in $\left(\mathscr{R}, \mathrm{V}, \Lambda^{r}\right)$.

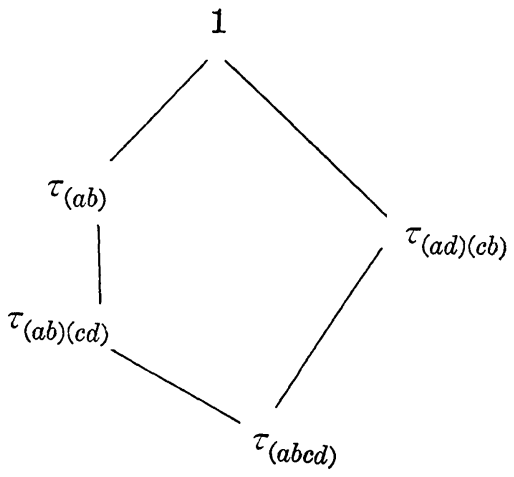

FIGURE 1

Greatest lower Bounds in $\mathscr{R}$ and continuous functions. In a paper in 1968 [14, p. 1087, Theorem 1], J. Pelham Thomas characterized the strongest regular topology on a set weaker than a given topology on that set: If $\tau$ is a topology on $E$, then there is a unique regular topology $\tau^{*}$ weaker than $\tau$, such that, if $Y$ is any regular space, then the continuous maps $(E, \tau) \rightarrow Y$ are the continuous maps $\left(E, \tau^{*}\right) \rightarrow Y$. Furthermore $\tau^{*}$ is the least upper bound of the regular topologies weaker than $\tau$. In this vein we have the following lemmas.

Lemma 2.1. A function $f:(E, 0) \rightarrow(Y, \rho)$ is continuous where $(Y, \rho)$ is a regular space iff $f(E) \subset \mathrm{cl}_{\rho}(f(x))$ for every $x \in E$.

Lemma 2.2. If, for every regular $T_{0}$ space $(Y, \rho)$, every continuous function $f:(E, \nu) \rightarrow(Y, \rho)$ is constant, then, for every regular space $(Y, \rho)$, every continuous function $f:(E, \nu) \rightarrow(Y, \rho)$ satisfies the condition $f(E) \subset \operatorname{cl}_{\rho}(f(x))$ for every $x \in E$.

Using the Thomas result we conclude that 
COROLlaRy 2.3. In order for $\sigma \Lambda^{r} \tau=0$ it is necessary and sufficient that every continuous function on $(E, \sigma \wedge \tau)$ to a regular $T_{0}$ space be constant.

It is now possible to reduce the problem to $T_{0}$ regular topologies. Let $\tau$ be a regular topology on $E$ and $E^{*}$ the set of point closures $\left\{\operatorname{cl}_{\tau}(x): x \in E\right\}$. Then $E^{*}$ is a set of equivalence classes of $E$ and $\varphi: E \rightarrow E^{*}$ given by $\varphi(x)=\operatorname{cl}_{\tau}(x)$ is the canonical map. If $\tau^{*}$ is the quotient topology relative to $\varphi$ and $\tau$, that is, the finest topology on $E^{*}$ such that $\varphi$ is continuous relative to $(E, \tau)$, then $\tau^{*}$ is a regular $T_{0}$ topology, lattice-isomorphic to $\tau$ [15, p. 92, Theorem 14.2]; further, $\varphi:(E, \tau) \rightarrow\left(E^{*}, \tau^{*}\right)$ is open and closed [9, p. 155, Theorem 9.3.6], and $\left(E^{*}, \tau^{*}\right)$ is called the $T_{0}$ quotient of $(E, \tau)$.

THEOREM 2.4. If the $T_{0}$ quotient $\left(E^{*}, \tau^{*}\right)$ of a regular space $(E, \tau)$ has a (principal) complement in the lattice of regular topologies on $E^{*}$, then $(E, \tau)$ has a (principal) complement in the lattice of regular topologies on $E$.

Proof. Let $f$ be a choice function on the subsets of $E, \sigma^{*}$ the regular complement for $\tau^{*}$ and $S=\left\{y \in E: y \neq f\left(\mathrm{cl}_{\tau}(y)\right)\right\}$. Define $\sigma$ to be the topology on $E$ with the following base

$$
\left\{\left(\varphi^{-1} B^{*}\right)-S: B^{*} \in \sigma^{*}\right\} \cup\{\{y\}: y \in S\} \text {. }
$$

The topology $\sigma$ is, in fact, regular. Suppose $F$ is closed in $(E, \sigma)$ and $x \notin F$. Then $\sim F=\left(\varphi^{-1} B^{*}-S\right) \cup A$ for some $A \subset S$ and some $B^{*} \in \sigma^{*}$. If $x \in S$, then $\{x\} \in \sigma$ and $F \subset E-\{x\} \in \sigma$. If $x \notin S$, then $\varphi x \in B^{*} \in \sigma^{*}$ and there exist disjoint sets $U^{*}, V^{*} \in \sigma^{*}$ separating $\varphi(x)$ and $\sim B^{*}$. In which case, $\varphi^{-1} U^{*}-S$ and $\varphi^{-1} V^{*} \cup S$ are $\sigma$-open sets separating $x$ and $F$. Note that $\sigma$ is principal if $\sigma^{*}$ is.

Next, if $A \in \sigma \wedge \tau$, then $\varphi A \in \tau^{*}$ and $A=\varphi^{-1} B^{*}$ for some $B^{*} \in \sigma^{*}$. Hence $\varphi:(E, \sigma \wedge \tau) \rightarrow\left(E^{*}, \sigma^{*} \wedge \tau^{*}\right)$ is open. If $\psi:(E, \sigma \wedge \tau) \rightarrow Y$ is any continuous function to a regular $T_{0}$ space $Y$, then $\psi\left(\mathrm{cl}_{\sigma \wedge}(x)\right)=\psi(x)$ for any $x \in E$. Hence $\psi \varphi^{-1}:\left(E^{*}, \sigma^{*} \wedge \tau^{*}\right) \rightarrow Y$ is a welldefined continuous function. Since $\sigma^{*} \Lambda^{r} \tau^{*}=0$ then $\psi \varphi^{-1}$ must be constant, which implies that $\psi$ is constant and hence $\sigma \Lambda^{r} \tau=0$.

Finally $\sigma \vee \tau=1$. For $x \notin S$ we have $U^{*} \in \tau^{*}$ and $V^{*} \in \sigma^{*}$ such that $\{\varnothing x\}=U^{*} \cap V^{*}$ which implies that

$$
\{x\}=\left(\varphi^{-1} U^{*}\right) \cap\left(\varphi^{-1} V^{*}-S\right) \in \tau \vee \sigma .
$$

Principal complementation and connectivity. In order for a regular topology $\tau$ and a principal regular topology $\sigma$ to have a least upper bound of 1 , it is necessary and sufficient that the minimal open 
sets of $\sigma$ be discrete in $\tau$. That they have a greatest lower bound of 0 is characterized in terms of continuous functions. Now a function is continuous on $(E, \sigma \wedge \tau)$ iff it is continuous on both $(E, \sigma)$ and $(E, \tau)$. Relative to continuity on principal regular spaces, we have the following:

Lemma 3.1. Let $\sigma$ be a principal regular topology on $E$. $A$ function $f:(E, \sigma) \rightarrow(Y, \rho)$, where $\rho$ is a $T_{1}$ topology, is continuous iff $f$ is constant on each minimal $\sigma$-open set.

THEOREM 3.2. If $(E, \tau)$ is a regular $T_{0}$ space with a disjoint open cover $\left\{E_{\alpha}\right\}_{\alpha}$ of $E$ and if, for each $\alpha$, the topology $\tau_{\alpha}=\tau \mid E_{\alpha}$ has a principal complement $\sigma_{\alpha}$ in the lattice of regular topologies on $E_{\alpha}$ then $\tau$ has a principal complement in the lattice of regular topologies on $E$.

Proof. For each $\alpha$ let $B^{\alpha}$ be some one minimal open set in $\sigma_{\alpha}$. The set $\mathrm{U}_{\alpha} B^{\alpha}$ and, for all $\alpha$, all minimal open sets $B_{x}$ in $\sigma_{\alpha}$, different from $B^{\alpha}$, define a minimal open base for a principal regular topology $\sigma$ on $E$ such that $\sigma \mid E_{\alpha}=\sigma_{\alpha}$.

Let $f$ be any function on $E$ to a regular $T_{0}$ space which is continuous relative to the topology $\sigma \wedge \tau$. Then for any $\alpha, f_{\alpha}=f \mid E_{\alpha}$ is continuous relative to the topology $(\sigma \wedge \tau) \mid E_{\alpha}$. But $(\sigma \wedge \tau) \mid E_{\alpha} \leqq$ $\sigma_{\alpha} \wedge \tau_{\alpha}$ so $f_{\alpha}$ is constant on $E_{\alpha}$. Since $f$ was continuous relative to $\sigma$ then $f$ must be constant on $\mathbf{U}_{\alpha} B^{\alpha}$. Hence $f$ is constant on all of $E$.

Lastly $\sigma \vee \tau=1$ : if $x$ is any point of $E=\bigcup_{\alpha} B_{\alpha}$ then $\sigma_{\alpha} \vee \tau_{\alpha}=$ 1 implies that there are sets $U \in \sigma$ and $V \in \tau$ such that $\{x\}=(U \cap$ $\left.E_{\alpha}\right) \cap\left(V \cap E_{\alpha}\right)=U \cap\left(V \cap E_{\alpha}\right) \in \sigma \vee \tau$.

The complementation problem for locally connected regular spaces is then reduced to the complementation problem for connected spaces. Further, the proof of the previous theorem suggests several lines of development.

THEOREM 3.3. Let $(E, \tau)$ be a regular $T_{0}$ space whose set $\mathscr{E}$ of components satisfy the following conditions:

(i) $\mathscr{E}$ is countable.

(ii) For each $C \in \mathscr{E}$ the restriction $\tau \mid C$ has a principal regular complement.

(iii) Either $\mathscr{E}$ has finitely many singletons or infinitely many nonsingletons.

Then $\tau$ has a principal regular complement.

Proof. Without loss of generality, by (i) the collection of com- 
ponents forms a sequence $\left\{E_{n}\right\}_{n}$ such that, by (iii) each singleton is followed by a nonsingleton. For each $n$, let $\tau_{n}=\tau \mid E_{n}$ and $\sigma_{n}$ its principal regular complement.

Now for any nonsingleton $E_{n}$ there must be at least two distinct minimal open sets in $\sigma_{n}$; otherwise $\tau_{n}=1$. But 1 is not connected unless $\left|E_{n}\right|=1$.

For each $n$, choose $A^{n}$ and $B^{n}$ minimal open sets in $\sigma_{n}$ such that $B^{n} \neq A^{n}$ if $\left|E_{n}\right|>1$. Then the sets

(i) $B^{n} \cup A^{n+1}$ for all $n$ such that $\left|E_{n}\right| \neq 1$ and $\left|E_{n+1}\right| \neq 1$

(ii) $B^{n} \cup E_{n+1} \cup A^{n+2}$ for all $n$ such that $\left|E_{n+1}\right|=1$

(iii) $B^{n-1} \cup E_{n} \cup A^{n+1}$ for all $n$ such that $\left|E_{n}\right|=1$

(iv) $B_{x}$ for all minimal $\sigma_{n}$ open sets with $B_{x} \neq A^{n}, B^{n}, n=1, \ldots$ define a base of minimal open sets for a principal regular topology $\sigma$ on $E$ such that $\sigma_{n}=\sigma \mid E_{n}$ for each $n$.

Let $f$ be any function on $E$ to a regular $T_{0}$ space which is continuous relative to the topology $\sigma \wedge \tau$. Then $f_{n}=f \mid E_{n}$ is continuous relative to the topology $\sigma_{n} \wedge \tau_{n}$ for each $n$. Hence $f_{n}$ is constant on $E_{n}$ and since $f$ is constant on each set in $\sigma$ then $f$ is constant on all of $E$.

For each $x$ not in some $B^{n}$ or $A^{n}$ there are sets $U \in \tau$ and $B_{x} \in \sigma_{n}$ such that $\{x\}=\left(U \cap E_{n}\right) \cap B_{x}=U \cap B_{x} \in \sigma \vee \tau$. For any $x \in B^{n}$ there is a neighborhood $U \in \tau$ of $x$ such that $U \cap B^{n}=\{x\}$ and, since components are closed and $x \notin E_{n \pm 1}, E_{n+2}$, such that $U \cap E_{n \pm 1}=\varnothing$ and $U \cap E_{n+2}=\varnothing$. Hence

$$
\begin{aligned}
\{x\} & =U \cap\left(B^{n} \cup A^{n+1}\right) \in \tau \vee \sigma \text { if }\left|E_{n}\right|,\left|E_{n+1}\right| \neq 1 ; \\
& =U \cap\left(B^{n} \cup E_{n+1} \cup A^{n+2}\right) \text { if }\left|E_{n+1}\right|=1 ; \\
& =U \cap\left(B^{n-1} \cup E_{n} \cup A^{n+1}\right) \text { if }\left|B_{n}\right|=\left|E_{n}\right|=1 .
\end{aligned}
$$

Similarly for any $x \in A^{n}$. Thus $\sigma \vee \tau=1$.

THEOREM 3.4. Let $(E, \tau)$ be a regular space and $D$ a dense subset. If $\tau \mid D$ has a complement $\sigma^{*}$ in the lattice of regular topologies on $D$, then $\tau$ has a complement in the lattice of regular topologies on $E$.

Proof. Define $\sigma$ to be the topology on $E$ with the base $\sigma^{*} \cup\{\{y\}$ : $y \notin D\}$. Then $\sigma$ is regular, $\sigma \mid D=\sigma^{*} ; \sigma$ is principal iff $\sigma^{*}$ is principal. Now clearly $(\sigma \wedge \tau)|D \leqq \sigma| D \wedge \tau \mid D$ so $\left(\sigma \wedge^{r} \tau\right)|D \leqq \sigma| D \Lambda^{r} \tau \mid D=0$. In which case, for any nonemply $U \in \sigma \Lambda^{r} \tau$ we have $U \supset D$ since $U \cap D=\varnothing$ is impossible. Hence $\sigma \Lambda^{r} \tau=0$. Obviously $\sigma \vee \tau=1$.

It is now clear that the complementation problem can be reduced to considering spaces without isolated points, because in the following result $(W, \tau \mid W)$ has no isolated points. 
COROLLARY 3.5. Let $(E, \tau)$ be a regular $T_{0}$ space, $I$ the set of isolated points, $W=$ int $_{\tau}(E-I)$ the interior of $E-I$. If $(W, \tau / W)$ has a principal regular complement then there is a principal regular complement for $\tau$.

Classes with complements. In this section our task is to construct principal regular complements for various classes of regular $T_{0}$ topologies. The first result provides the basic construction used in the following theorem to handle the class of supra- $D N$ spaces. The definition of this class is a generalization of the $D N$ spaces of $\mathrm{B}$. A. Anderson [1, p. 989] and was suggested by Harold Bell as a means of extending methods developed for the $D N$ spaces. The question remains open whether this class exhausts the regular $T_{0}$ spaces. Subsequent results show an approach to a different class of spaces and to arbitrary products of such spaces.

THEOREM 4.1. Let $(E, \tau)$ be a regular $T_{0}$ space, $\xi>|E|$, and $\left\{S_{n}: 0 \leqq n<\eta \leqq \xi\right\}$ a wellordered family of disjoint discrete nonempty subsets of $E$ whose union is dense in $E$. Suppose that for such $n>0$, any open set containing $\mathrm{cl}_{\tau}\left(\mathbf{U}_{r<n} S_{r}\right)$ meets $S_{n}$. Then $\tau$ has a principal regular complement $\sigma$. Moreover there is some point $x \in E$ such that $\mathrm{cl}_{\sigma \wedge \tau}(x)=E$.

Proof. Define $\sigma$ to be the principal regular topology with the base of minimal open sets $\left\{S_{n}: n \geqq 0\right\} \cup\left\{\{x\}: x \notin \mathbf{U}_{n \geqq 0} S_{n}\right\}$. Then for any $x \in E$ we have $\{x\} \in \sigma \vee \tau$.

On the other hand, for each $S_{n}$ let $x_{n}$ be any point in $\operatorname{cl}_{\tau}\left(S_{n}\right)$. Suppose there is an ordinal $n$ such that

$$
\mathrm{cl}_{\sigma \wedge r_{\tau}}\left(x_{n}\right) \neq \mathrm{cl}_{\sigma \wedge r_{\tau}}\left(x_{0}\right) \text {. }
$$

Let $m$ be the least such ordinal. Then there are disjoint sets $U^{*}$, $V^{*} \in \sigma \Lambda^{r} \tau$ such that $\operatorname{cl}_{\sigma \wedge r_{\tau}}\left(x_{0}\right) \subset U^{*}$ and $\operatorname{cl}_{\sigma \wedge r_{\tau}}\left(x_{m}\right) \subset V^{*}$. Also, for every $\gamma<m, \mathrm{cl}_{\sigma \wedge r_{\tau}}\left(x_{0}\right)=\operatorname{cl}_{\sigma \wedge r_{\tau}}\left(x_{\gamma}\right)$. But then $\mathrm{cl}_{\sigma \wedge r_{\tau}}\left(x_{0}\right)$ is a $\tau$-closed set containing all the sets $\mathrm{cl}_{\sigma \wedge r_{\tau}}\left(x_{\gamma}\right) \supset S_{\gamma}$ for $\gamma<m$. By the regularity, every $U \in \sigma \wedge^{r} \tau$ such that $x_{0} \in U$ must contain $\operatorname{cl}_{\sigma \wedge r_{\tau}}\left(x_{0}\right) \supset \operatorname{cl}_{\tau}\left(\mathbf{U}_{\gamma<m} S_{\gamma}\right)$. So $U^{*}$ meets $S_{m} \subset \mathrm{cl}_{\sigma \wedge r_{\tau}}\left(x_{m}\right) \subset V^{*}$ which is a contradiction. Hence $\mathrm{cl}_{\sigma \wedge r_{\tau}}\left(x_{0}\right)=E$ and $\sigma \Lambda^{r} \tau=0$.

Definition. A space $(E, \tau)$ is said to be supra- $D N$ if, for any open set $U$ such that $\operatorname{cl}_{\tau}(U)-U \neq \varnothing$ there is a discrete set $S \subset U$ such that $\operatorname{cl}_{r}(S)-U \neq \varnothing$.

Note that any first countable space is supra- $D N$.

THEOREM 4.2. If $(E, \tau)$ is a regular $T_{0}$ supra-DN space without 
isolated points then $\tau$ has a principal regular complement.

Proof. Let $x_{1}$ be any point of $E$ and $U_{1}=E-\left\{x_{1}\right\} \in \tau$. Then there is a discrete set $S_{1} \subset U_{1}$ such that $\left\{x_{1}\right\}=\operatorname{cl}_{\tau}\left(S_{1}\right)-U_{1}$. For the induction, consider any ordinal $n$ between 1 and $\xi$, where $\xi>|E|$; suppose that for each $\beta<n$ the set $S_{\beta} \subset E-\operatorname{cl}_{\tau}\left(\bigcup_{r<\beta} S_{\gamma}\right)$ is defined, nonclosed, discrete, and either $\operatorname{cl}_{\tau}\left(\bigcup_{r<\beta} S_{\gamma}\right) \in \tau$ or any open set containing $\operatorname{cl}_{\tau}\left(\bigcup_{r<\beta} S_{\gamma}\right)$ meets $S_{\beta}$. Now for any subset $A \subset E$, either the boundary of $E-\operatorname{cl}_{\tau}(A)$ is nonempty or $\operatorname{cl}_{\tau}(A)$ is open. Hence if $\operatorname{cl}_{\tau}\left(\mathbf{U}_{\gamma<n} S_{\gamma}\right)$ is not open then the boundary of $U_{n}=E-\operatorname{cl}_{\tau}\left(\mathbf{U}_{\tau<n} S_{\gamma}\right) \in \tau$ contains some point $x_{n}$ and $U_{n}$ contains a discrete set $S_{n}$ such that $x_{n} \in \mathrm{cl}_{\tau}\left(S_{n}\right)-U_{n}$. So any open set containing $\mathrm{cl}_{\tau}\left(\mathrm{U}_{r<n} S_{\gamma}\right)$ contains the boundary of $U_{n}$ and hence, as a neighborhood of $x_{n}$, meets $S_{n}$. If, on the other hand, $\operatorname{cl}_{\tau}\left(\bigcup_{r<n} S_{r}\right) \in \tau$, let $x_{n}$ be any point of $V_{n}=$ $E-\operatorname{cl}_{\tau}\left(\bigcup_{r<n} S_{\gamma}\right)$ and $U_{n}=V_{n}-\left\{x_{n}\right\} \in \tau$. Then there is a discrete set $S_{n} \subset U_{n}$ such that $\left\{x_{n}\right\}=\operatorname{cl}_{\tau}\left(S_{n}\right)-U_{n}$.

Consequently $\operatorname{cl}_{\tau}\left(\bigcup_{1 \leqq n} S_{n}\right)=E$ and $S_{0}=\left\{x_{n}: \operatorname{cl}_{\tau}\left(\bigcup_{r<n} S_{\gamma}\right) \in \tau\right\}$ is discrete. Lastly, if $\mathrm{cl}_{\tau}\left(\bigcup_{1 \leqq r<n} S_{r}\right) \in \tau$ then any $\tau$-open set containing $\operatorname{cl}_{\tau}\left(\mathrm{U}_{0 \leqq r<n} S_{\gamma}\right) \supset S_{0}$, and hence containing $x_{n}$, meets $S_{n}$. Otherwise $\operatorname{cl}_{\tau}\left(\mathbf{U}_{1 \leq \gamma<n} S_{\gamma}\right) \notin \tau$ and any $U \in \tau$ such that $U \supset \operatorname{cl}_{\tau}\left(\mathbf{U}_{0 \leqq \gamma<n} S_{\gamma}\right)$ must meet $S_{n}$. The conclusion then follows by the previous theorem.

Definition. A space $(E, \tau)$ is said to be Bolzano-Weierstrass compact if every infinite subset of $E$ has a limit point in $E$.

Definition. A space $(E, \tau)$ is said to be locally-B.W.-compact if each point in the space has a fundamental system of neighborhoods each of which is Bolzano-Weierstrass compact.

THEOREM 4.3. If $(E, \tau)$ is a separable, regular $T_{0}$ locally-B.W.compact space without isolated points, then $\tau$ has a principal regular complement.

Proof. Let $Q=\left\{q_{1}, q_{2}, \cdots\right\}$ be a countable dense subset of $E$. Let $V_{1}$ be a B.W. compact neighborhood of $x_{1}=q_{1}$. Since $\tau \mid Q$ is $T_{2}$ without isolated points, there is a countably infinite discrete $S_{1} \subset$ int $_{\tau}\left(V_{1}\right) \cap Q$ with $x_{1} \in S_{1}$. For every $x \in S_{1}$, the $T_{2}$ regularity of $E$ and the discreteness of the countable set $S_{1}$ imply that there is an open set $V_{x}$ such that $x \in V_{x} \subset \mathrm{cl}_{\tau} V_{x} \subset V_{1}, \mathrm{cl}_{\tau} V_{x} \cap \mathrm{cl}_{\tau} S_{1}=\{x\}$, and if $x, y \in S_{1}$ and $x \neq y$, then $\operatorname{cl}_{\tau} V_{x} \cap \operatorname{cl}_{\tau} V_{y}=\varnothing$. Hence, for each $x \in S_{1}$, an infinite discrete set $S_{x}$ may be chosen so that $x \in S_{x} \subset V_{x} \cap Q$.

The points of $S_{1}$ may be denoted by $x_{1 n}$ for $n=1,2, \cdots$, with $x_{11}=x_{1}$. The corresponding discrete sets may be denoted by $S_{1 n}$. For each $n$, let $y_{1 n} \in \operatorname{cl}_{\tau}\left(S_{1 n}\right)-S_{1 n} \subset \operatorname{cl}_{\tau} V_{x_{1 n}}$. 
For each $k>1$ let $Q_{k}=Q-\operatorname{cl}_{\tau}\left(\mathbf{U}_{p<k} \mathbf{U}_{n=1}^{\infty} S_{p n}\right) \in \tau \mid Q$. If $Q_{k} \neq \varnothing$, let $x_{k}$ be the least element in the order on $Q_{k}$.

$V_{k}$ a B.W.-compact neighborhood of $x_{k}$ in $\sim \mathrm{cl}_{\tau}\left(\mathbf{U}_{p<k} \bigcup_{n=1}^{\infty} S_{p n}\right)$

$S_{k}$ a countably infinite discrete set in $V_{k} \cap Q_{k}$ with $x_{k} \in S_{k}$

$x_{k n} n=1,2, \cdots$ the points of $S_{k}$ in the induced order

$S_{k n}$ the corresponding countably infinite discrete sets chosen from the intersection of $Q$ and a neighborhood, of $x_{k n}$, whose closure is in $V_{k}$ with $x_{k 1}=x_{k} \in S_{k 1}$ and satisfying $\operatorname{cl}_{\tau} S_{k n} \cap \operatorname{cl}_{\tau} S_{k p}=\varnothing$ for $n \neq p$, and $y_{k n} \in \operatorname{cl}_{\tau}\left(S_{k n}\right)-S_{k n}$.

Clearly $\operatorname{cl}_{\tau}\left(\bigcup_{p=1}^{\infty} \bigcup_{n=1}^{\infty} S_{p n}\right) \supset \operatorname{cl}_{\tau}(Q)=E$.

Define a principal regular complement $\sigma$ for $\tau$ with a base of minimal open sets consisting of

$$
\begin{array}{rlrl}
U_{1} & =S_{11}, & \\
U_{k} & =S_{1 k} \cup\left\{y_{1(k-1)}\right\} \cup S_{k 1} & & \text { for } k>1, \\
U_{p k} & =S_{p k} \cup\left\{y_{p(k-1)}\right\} & & \text { for } p, k>1, \\
\{y\} & & \text { for all } y \notin\left(\bigcup_{k} U_{k}\right) \cup\left(\bigcup_{p, k} U_{p k}\right) .
\end{array}
$$

The minimal open sets are discrete in $(E, \tau)$ because $S_{k 1}$ was chosen in a closed neighborhood outside $\operatorname{cl}_{\tau}\left(\bigcup_{p<k} \bigcup_{m=1}^{\infty} S_{p m}\right)$ which contains $\mathrm{cl}_{\tau}\left(S_{1 k}\right)$, and because $y_{n(k-1)} \in \mathrm{cl}_{\tau}\left(S_{n(k-1)}\right)$ and $\mathrm{cl}_{\tau}\left(S_{n(k-1)}\right) \cap \mathrm{cl}_{\tau}\left(S_{n k}\right)=\varnothing$.

Lastly, if $U \in \tau \wedge \sigma, U \neq \varnothing$, then $U \cap\left(\bigcup_{p, k} S_{p k}\right) \neq \varnothing$. Let $\bar{\alpha}$ be the least ordinal for which there is a $\beta$ such that $U \cap S_{\bar{\alpha} \beta} \neq \varnothing$ and $\bar{\beta}$ the least such $\beta$. Suppose $\bar{\alpha} \neq 1$. Then $\bar{\beta} \neq 1$ and $y_{\bar{\alpha}(\bar{\beta}-1)} \in U_{\bar{\alpha} \bar{\beta}} \subset$ $U \in \sigma$. But $y_{\bar{\alpha}(\bar{\beta}-1)}$ is a $\tau$-limit point of $S_{\bar{\alpha}(\bar{\beta}-1)}$ so $U \in \tau$ meets $S_{\bar{\alpha}(\bar{\beta}-1)}$ which contradicts the minimality of $\bar{\beta}$. Hence $\bar{\alpha}=1$. Similarly $\bar{\beta}=1$ and $S_{11}=U_{1} \subset U$ for every $U \in \tau \wedge \sigma$ and $\sigma \Lambda^{r} \tau=0$.

Note that local compactness and countable compactness imply local-B.W.-compactness.

THEOREM 4.4. For each $i \in \theta$ let $\left(E_{i}, \tau_{i}\right)$ be a regular $T_{0}$ space for which there exists a principal regular topology $\sigma_{i}$ on $E_{i}$ such that

(a) $\sigma_{i} \vee \tau_{i}=1$.

(b) There is a subset $W_{i} \subset E_{i}$ such that $U \in \sigma_{i} \wedge \tau_{i}$ and $U \neq \varnothing$ imply that $U \supset W_{i}$.

(c) If $U \in \tau_{i}$ satisfies $U \supset W_{i}$ then there are $\sigma_{i}$-isolated points in $U$.

(d) The set of $\sigma_{i}$-nonisolated points is dense in $\left(E_{i}, \tau_{i}\right)$. If $E=\prod_{i \in \theta} E_{i}$ and $\tau=\prod_{i \in \theta} \tau_{i}$ then $(E, \tau)$ has a principal regular complement.

Proof. Well order $\theta$; let $\left(x_{i}\right)_{i} \in E$. If $x_{i}$ is isolated in $\sigma_{i}$ for every $i \in \theta$, then let $B\left(x_{i}\right)_{i}=\left\{\left(x_{i}\right)_{i}\right\}$. Otherwise, there is a least element $\bar{i} \in \theta$ such that $x_{i}$ is not $\sigma_{i}$-isolated; let $B\left(x_{i}\right)_{i}=B_{i} \times\left(x_{i}\right)_{i \neq \tau}$ where $B_{i}$ 
is the minimal $\sigma_{i}$-open set containing $x_{i \cdot}$ The collection $\left\{B\left(x_{i}\right)_{i}:\left(x_{i}\right)_{i} \in\right.$ $E$ \} forms a base of minimal open sets for a principal regular topology $\sigma$ on $E$.

Using hypothesis (a) for the first nonisolated coordinate, it is easily seen that $\sigma \vee \tau=1$.

Next let $A^{1}, A^{2} \in \sigma \wedge \tau$ be nonempty. Now $A^{1}, A^{2} \in \tau$ implies that there are indices $i_{1}, i_{2}, \cdots, i_{k} \in \theta$ such that $A^{1}$ and $A^{2}$ contain rectangular neighborhoods. Hence there are points $\left(x_{i}\right)_{i} \in A^{1}$ and $\left(y_{i}\right)_{i} \in A^{2}$ such that $x_{i}=y_{i}$ for $i \neq i_{1}, \cdots, i_{k}$ and, by $(d), x_{i}, y_{i}$ are $\sigma_{i}$-nonisolated for $i=i_{1}, \cdots, i_{k}$ only. Let $j=\min \left\{i_{1}, \cdots, i_{k}\right\}$ and $A_{j}^{1}=\left\{z \in E_{j}:\{z\} \times\right.$ $\left.\left(x_{i}\right)_{i \neq j} \in A^{1}\right\} \in \tau_{j}$, the inverse image of $A^{1}$ under the $\left(x_{i}\right)_{i \neq j}$-section; since $x_{i}$ is $\sigma_{i}$-isolated for $i<j$ then for any $\sigma_{j}$-nonisolated point $x \in A_{j}^{1}$, $B_{x} \times\left(x_{i}\right)_{i \neq j} \subset A^{1}$. In which case, $B_{x} \subset A_{j}^{1}$ and hence $A_{j}^{1} \in \sigma_{j}$. Similarly $A_{j}^{2}=\left\{z \in E_{j}:\{z\} \times\left(y_{i}\right)_{i \neq j} \in A^{2}\right\} \in \sigma_{j} \wedge \tau_{j}$. Thus by (b), $W_{j} \subset A_{j}^{1} \cap A_{j}^{2} \in \sigma_{j} \wedge \tau_{j}$ and by (c), there is an isolated point $x_{j}^{\prime}=y_{j}^{\prime}$ in $A_{j}^{1} \cap A_{j}^{2}$ which means that

$$
\left(x_{i}\right)_{i \neq j} \times x_{j}^{\prime} \in A^{1} \text { and }\left(y_{i}\right)_{i \neq j} \times y_{j}^{\prime} \in A^{2} .
$$

Continuing this process and replacing $x_{i_{1}}, \cdots, x_{i_{k}}$ and $y_{i_{1}}, \cdots, y_{i_{k}}$ locates a point common to $A^{1}$ and $A^{2}$. The absence of disjoint sets in $\tau \wedge \sigma$ implies that $\tau \Lambda^{r} \sigma=0$.

In particular, the principal regular complement constructed in Theorem 4.3 satisfies conditions (a), (b) and (d) required of the factor spaces in Theorem 4.4; condition (c) can be accomodated without losing others.

\section{REFERENCES}

1. B. A. Anderson, A class of topologies with $T_{1}$ complements, Notices Amer. Math. Soc., 16 (1969), 69T-G141, 989.

2. B. A. Anderson and D. G. Stewart, $T_{1}$ complements of $T_{1}$ topologies, Proc. Amer. Math. Soc., 23 (1970), 77-81.

3. R. W. Bagley, On the characterization of the lattice of topologies, J. London Math. Soc., 30 (1955), 247-249.

4. N. Bourbaki, Topologie Generale, Hermann, Paris, 1961.

5. O. Frohlich, Das Halbordnunssystem der topologischen Raume auf einer Mange, Math. Ann., 156 (1964), 79-95.

6. H. Gaifman, The lattice of all topologies, Canad. J. Math., 18 (1966), 83-85.

7. J. Hartmanis, On the lattice of topologies, Canad. J. Math., 10 (1958), 547-553.

8. M. J. Norris, A note on regular and completely regular topological spaces, Proc. Amer. Math. Soc., 1 (1950), 754-755.

9. W. J. Pervin, Foundations of General Topology, Academic Press, New York, 1964. 10. A. K. Steiner, The lattice of topologies: structure and complementation, Trans. Amer. Math. Soc., 122 (1966), 379-398.

11. Complementation in the lattice of $T_{1}$ topologies, Proc. Amer. Math. Soc., 17 (1967), 884-886.

12. A. K. Steiner and E. F. Steiner, Topologies with $T_{1}$ complements, Fund. Math., 61 (1967), 23-28. 
13. $A T_{1}$ complement for the reals, Proc. Amer. Math. Soc., 19 (1968) 177-179. 14. J. P. Thomas, Associated regular spaces, Canad. J. Math., 20 (1968), 1087-1092. 15. W. J. Thron, Topological Structures, Holt Rinehart Winston, New York, 1966.

16. A. C. M. van Rooij, The lattice of all topologies is complemented, Canad. J. Math., 20 (1968), 805-807.

Received July 15, 1971 and in revised form September 6, 1971. 



\section{PACIFIC JOURNAL OF MATHEMATICS}

\section{EDITORS}

\section{H. SAMElson}

Stanford University

Stanford, California 94305

C. R. Новву

University of Washington

Seattle, Washington 98105
J. DugunduI

Department of Mathematics University of Southern California

Los Angeles, California 90007

RICHARD ARENS

University of California

Los Angeles, California 90024

\section{ASSOCIATE EDITORS}
E. F. BECKENBACH
B. H. NeUmanN
F. WOLF
K. YosHIDA

\section{SUPPORTING INSTITUTIONS}

UNIVERSITY OF BRITISH COLUMBIA

UNIVERSITY OF SOUTHERN CALIFORNIA

CALIFORNIA INSTITUTE OF TECHNOLOGY

STANFORD UNIVERSITY

UNIVERSITY OF CALIFORNIA

UNIVERSITY OF TOKYO

MONTANA STATE UNIVERSITY

UNIVERSITY OF UTAH

UNIVERSITY OF NEVADA

WASHINGTON STATE UNIVERSITY

NEW MEXICO STATE UNIVERSITY

OREGON STATE UNIVERSITY

UNIVERSITY OF OREGON

OSAKA UNIVERSITY

UNIVERSITY OF WASHINGTON

* *

AMERICAN MATHEMATICAL SOCIETY

NAVAL WEAPONS CENTER 


\section{Pacific Journal of Mathematics}

\section{Vol. 43, No. $1 \quad$ March, 1972}

Alexander (Smbat) Abian, The use of mitotic ordinals in cardinal

arithmetic ....................................... 1

Helen Elizabeth. Adams, Filtrations and valuations on rings ......... 7

Benno Artmann, Geometric aspects of primary lattices .............. 15

Marilyn Breen, Determining a polytope by Radon partitions ........... 27

David S. Browder, Derived algebras in $L_{1}$ of a compact group .......... 39

Aiden A. Bruen, Unimbeddable nets of small deficiency .............. 51

Michael Howard Clapp and Raymond Frank Dickman, Unicoherent

compactifications ............................... 55

Heron S. Collins and Robert A. Fontenot, Approximate identities and the strict topology ................................... 63

R. J. Gazik, Convergence in spaces of subsets................. 81

Joan Geramita, Automorphisms on cylindrical semigroups ........... 93

Kenneth R. Goodearl, Distributing tensor product over direct product ..... 107

Julien O. Hennefeld, The non-conjugacy of certain algebras of

operators ................................... 111

C. Ward Henson, The nonstandard hulls of a uniform space ........... 115

M. Jeanette Huebener, Complementation in the lattice of regular

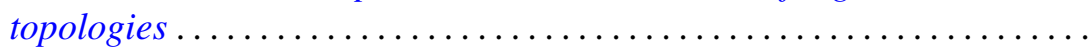

Dennis Lee Johnson, The diophantine problem $Y^{2}-X^{3}=A$ in a

polynomial ring .................................... 151

Albert Joseph Karam, Strong Lie ideals . . . . . . . . . . . . . . . . . . . . 157

Soon-Kyu Kim, On low dimensional minimal sets ............... 171

Thomas Latimer Kriete, III and Marvin Rosenblum, A Phragmén-Lindelöf

theorem with applications to $M(u, v)$ functions ..... . .

William A. Lampe, Notes on related structures of a universal algebra . . . . 189

Theodore Windle Palmer, The reducing ideal is a radical .

207

Kulumani M. Rangaswamy and N. Vanaja, Quasi projectives in abelian and module categories ................................ 221

Ghulam M. Shah, On the univalence of some analytic functions ......... 239

Joseph Earl Valentine and Stanley G. Wayment, Criteria for Banach

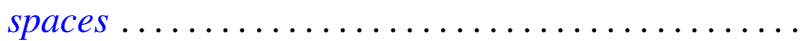

Jerry Eugene Vaughan, Linearly stratifiable spaces ............... 253

Zbigniew Zielezny, On spaces of distributions strongly regular with respect to partial differential operators ..................... 